\title{
Little evidence for association of the glaucoma gene MYOC with open-angle glaucoma
}

\author{
Seongsoo Sohn, ${ }^{1}$ Wonhee Hur, ${ }^{2,3}$ Young Ran Choi, ${ }^{2}$ Yun Shin Chung, ${ }^{4}$ \\ Chang-Seok Ki, ${ }^{5}$ Changwon Kee ${ }^{1}$
}

${ }^{1}$ Department of Ophthalmology, Samsung Biomedical Research Institute, Sungkyunkwan University School of Medicine, Seoul, South Korea

${ }^{2}$ Center for Clinical Research, Samsung Biomedical Research Institute, Sungkyunkwan University School of Medicine, Seoul, South Korea

${ }^{3}$ Present address: Department of Internal Medicine \& WHO Collaborating Center of Viral Hepatitis, The Catholic University of Korea, Seoul, 137-701, South Korea

${ }^{4}$ Transplantation Center,

Samsung Biomedical Research Institute, Sungkyunkwan University School of Medicine, Seoul, South Korea

5 Laboratory Medicine \& Cytogenetics, Samsung Medical Center, Sungkyunkwan University School of Medicine, Seoul, South Korea

\section{Correspondence to} Dr Changwon Kee, Department of Ophthalmology, Samsung Medical Center, Sungkyunkwan University School of Medicine, Seoul 135-710, South Korea; ckee@skku.edu

Accepted 29 November 2009

\section{ABSTRACT}

Background/aim To determine if overexpression of the glaucoma gene MYOC is involved in the development of open-angle glaucoma (OAG) and if its promoter variants are associated with glaucoma in the Korean population. Methods Human trabecular meshwork cells were cultured in the presence of ophthalmic steroids such as fluorometholone, fluorometholone acetate, dexamethasone, prednisolone acetate and rimexolone. The cells were cultured at a hydrostatic pressure of $32 \mathrm{~mm} \mathrm{Hg}$ above atmospheric pressure and induction of MYOC was evaluated by northern blot analysis. Genomic DNA was extracted from blood samples obtained from 74 normal controls and 168 unrelated Korean patients with $O A G$, including primary $O A G$, normal tension glaucoma and steroid-induced glaucoma. A 461 base pair (bp) DNA fragment of the MYOC promoter region was amplified using PCR and its genotype was analysed by directly sequencing the product.

Results The potencies of steroid eye drops in MYOC induction in vitro was the same regardless of their potential for elevating intraocular pressure in vivo. Hydrostatic pressure had no effect on MYOC induction. A dinucleotide repeat polymorphism and three single nucleotide polymorphisms were identified, but no obvious differences in the genotype distribution and allele frequency of the variants between the control group and any type of OAG were observed.

Conclusion Our data suggest that MYOC overexpression is not a cause or an effect of intraocular pressure elevation and that MYOC itself is not associated with $\mathrm{OAG}$.

\section{INTRODUCTION}

MYOC encodes a secretory glycoprotein of 504 amino acids named myocilin, and is the first gene to be linked to juvenile open-angle glaucoma (OAG) and some forms of adult-onset primary open-angle glaucoma (POAG). ${ }^{1}$

The gene was identified as an upregulated molecule in cultured trabecular meshwork (TM) cells after treatment with dexamethasone (DEX) and was originally referred to as trabecular meshworkinducible glucocorticoid response $(T I G R){ }^{2}$

It is well known that the long-term use of topical ophthalmic steroids results in ocular hypertension with glaucoma, known as steroid-induced glaucoma (SIG), in up to $40 \%$ of the population. ${ }^{3}$ SIG mimics many aspects of POAG and both diseases have been shown to be caused by elevated intraocular pressure (IOP) due to decreased aqueous outflow. Interestingly, the profile of MYOC upregulation by DEX is in a dose- and time-dependent manner very similar to the course of development of SIG. ${ }^{2}$ This led many investigators to believe that an increased MYOC level is a cause of glaucoma. ${ }^{4}$ However, a putative association between MYOC induction and OAG has not been firmly established. ${ }^{6-8}$

Determining whether or not $M Y O C$ expression can cause glaucoma is important for understanding the pathology of OAG for patients without mutations in the $M Y O C$ coding region and for assessing the risk of the disease with variants in the MYOC non-coding region. Therefore, in the present study, we investigated whether MYOC induction is involved in the development of glaucoma. We evaluated the potency of MYOC induction by ophthalmic steroids with different abilities to elevate IOP. We also investigated whether promoter variants of $M Y O C$ are associated with glaucoma.

\section{MATERIALS AND METHODS Cell culture}

Human trabecular meshwork (HTM) cells were maintained in Dulbecco's modified Eagle's medium (DMEM; Life Technologies, Grand Island, New York, USA) supplemented with $10 \%$ fetal bovine serum and antibiotics at $37^{\circ} \mathrm{C}$ in a humidified $5 \%$ $\mathrm{CO}_{2}-95 \%$ air atmosphere. The cells, generously provided by Paul L. Kaufman (University of Wisconsin, Madison, Wisconsin, USA), were derived from the normal eyes of a 27-year-old female donor.

\section{Steroid treatment}

HTM cells plated in $60-\mathrm{mm}$ culture dishes were grown to confluence before addition of steroids. All steroid eye drops (steroid ophthalmic suspensions) were purchased from Alcon Pharmaceuticals (Fort Worth, Texas, USA) and were prepared in phosphate buffered saline at $2.5 \mu \mathrm{M}$. Confluent HTM cells received doses of the diluted steroids and were cultured for up to 9 days, and the media were changed once every 3 days.

\section{Hydrostatic pressure}

Dishes with confluent HTM cells were placed at the bottom of a 2-1 mass cylinder (Nalgen, Rochester, New York, USA) in which fresh media had been poured up to a height of $43 \mathrm{~cm}$ from the bottom. The cells were cultured for 9 days in this condition, which was equivalent to a hydrostatic pressure of $32 \mathrm{~mm} \mathrm{Hg}$ above atmospheric pressure.

\section{Northern blot analysis}

At the end of culture, the HTM cells were harvested and total RNA was isolated. Approximately $5 \mu \mathrm{g}$ of 
total RNA was size-fractionated, transferred to a nylon membrane, and then hybridised at $65^{\circ} \mathrm{C}$ overnight with a ${ }^{32} \mathrm{P}$-labelled probe for human MYOC. After hybridisation, the membrane was washed twice in $2 \times$ (sodium chloride/sodium citrate)SSC $-0.1 \%$ (sodium dodecyl sulfate)SDS and once in $1 \times$ SSC $-0.1 \%$ SDS and was then autoradiographed at $-70^{\circ} \mathrm{C}$.

\section{Blood samples}

Peripheral blood samples were collected from 168 unrelated Korean glaucoma patients who visited the Department of Ophthalmology at Samsung Medical Center, Seoul, South Korea. For inclusion of POAG, the patients had to meet the following criteria. (1) An untreated mean IOP $>21 \mathrm{~mm} \mathrm{Hg}$ by Goldmann applanation tonometry. (2) The anterior chamber angles on gonioscopy were wide open. (3) The best corrected visual acuity was $20 / 30$ or better. (4) A typical glaucomatous optic nerve appearance including focal or generalised narrowing or disappearance of the neuroretinal rim, asymmetry in the cup to disc ratio $>0.2$, disc haemorrhage or retinal nerve fibre layer defects. (5) A glaucoma hemifield defect corresponding to the glaucomatous optic nerve damage detected in two consecutive fields, at intervals of at least 2 months apart. Normal tension glaucoma (NTG) was defined as for POAG, except for untreated mean IOP being $\leq 21 \mathrm{~mm} \mathrm{Hg}$ by Goldmann applanation tonometry. SIG was defined as OAG associated with a high IOP from administration of steroid eye drops for more than 2 weeks. Blood samples from a control group of 74 normal healthy Korean volunteers, unmatched for age or sex, were also obtained.

\section{Genotyping}

A 461 base pair (bp) DNA fragment of the $5^{\prime}$-flanking region of MYOC, containing sequences from $396 \mathrm{bp}$ upstream of the transcriptional initiation site to 65 bp of the untranslated region, was amplified from the genomic DNA by PCR with primers designed based on available sequence data (GenBank accession no. Z97171). The genotype distribution of polymorphisms was determined by direct sequencing of the PCR product with the terminator cycle-sequencing method using fluorescent dideoxynucleotides and an automatic DNA sequencer (Applied Biosystems, Foster City, California, USA). Statistical analysis was performed using the Pearson $\chi^{2}$ test.

\section{RESULTS}

Effect of steroids and hydrostatic pressure on MYOC induction Northern blot hybridisation revealed that the treatment of confluent HTM cells with DEX led to an apparent induction of MYOC gene expression (figure 1B). This result demonstrates that the HTM cells used in the present study were authentic because steroid-induced MYOC induction has been observed only in cultured TM cells or in TM tissues from perfused organ cultures. $^{2} 6$

The exposure of HTM cells to steroid eye drops also led to $M Y O C$ induction (figure 2). Treatment of cells with eye drops containing increasing concentrations of fluorometholone (Flucon), fluorometholone acetate (Flarex), DEX (Maxidex), prednisolone acetate (Pred forte) and rimexolone (Vexol) all induced MYOC expression in a dose-dependent manner. However, no differences were observed in the potency of these drugs in terms of MYOC induction. Time-course studies of MYOC induction also gave the same results of no differences in the steady-state levels of $M Y O C$ expression at any time points in cells treated with different steroids (data not shown).

A hydrostatic pressure equivalent to $32 \mathrm{~mm} \mathrm{Hg}$ above atmospheric pressure appeared to affect the morphology of the

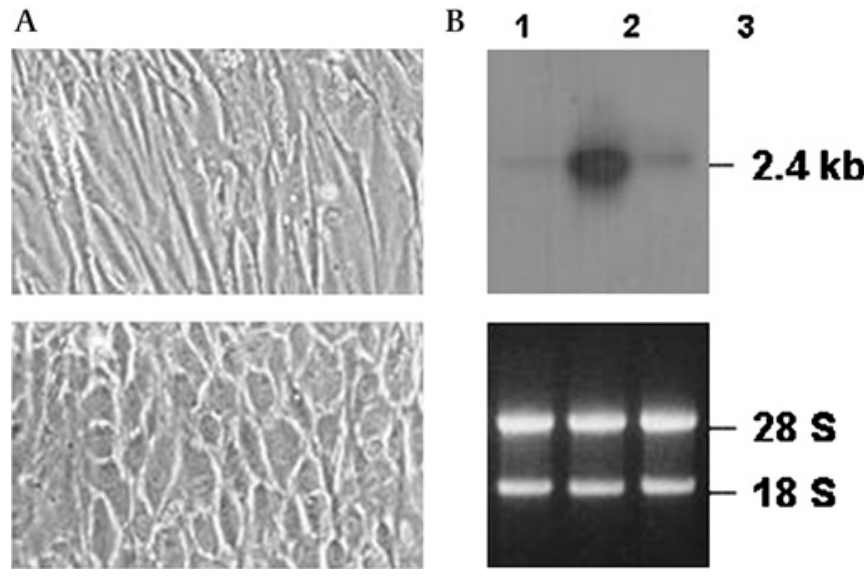

Figure 1 Effect of hydrostatic pressure on morphology and induction of MYOC expression in cultured human trabecular meshwork (HTM) cells.

(A) Phase-contrast images were photographed from cells cultured for 9 days in the absence (upper panel) or presence of a hydrostatic pressure of $32 \mathrm{~mm} \mathrm{Hg}$ above atmospheric pressure (lower panel). (B) RNA was extracted from cells cultured in the absence (lane 1) or presence of hydrostatic pressure (lane 3), hybridised with ${ }^{32} \mathrm{P}$-labelled MYOC cDNA, and subjected to autoradiography (upper panel). The same RNA was visualised by ethidium bromide staining (lower panel). Cells treated with $100 \mathrm{nM}$ dexamethasone (DEX) (lane 2) served as a positive control.

HTM cells (figure 1A). In addition to an increase in cell and nuclear size, it was evident that the original partially elongated shape changed to a rounder form. However, this morphological change was not accompanied by any prominent induction of MYOC (figure 1B).

\section{Association of MYOC with glaucoma}

Six alleles, ranging from 12 to 17 repeats of (G-T)n dinucleotides, were found in the microsatellite that had previously been described as being located 314 bp upstream from the translation start site of $M Y O C .^{2}$ Each allele was designated as $(\mathrm{G}-\mathrm{T})_{12}$ to (G$\mathrm{T})_{17}$ according to the number of repeats. The genotype distribution and allele frequencies of the $(\mathrm{G}-\mathrm{T})_{\mathrm{n}}$ microsatellite marker
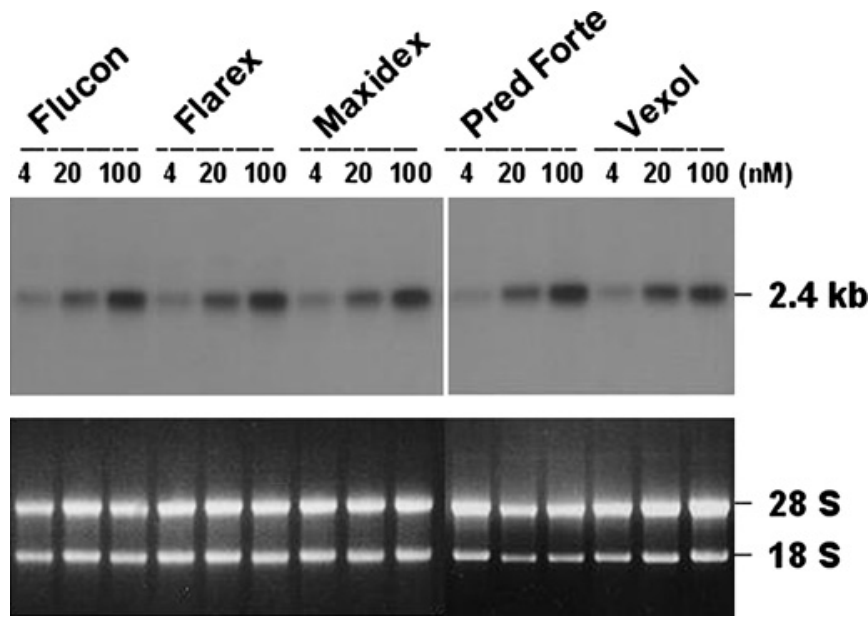

Figure 2 Effect of ophthalmic steroids on the induction of MYOC expression in cultured human trabecular meshwork (HTM) cells. Confluent HTM cells were cultured for 9 days in the presence of each steroid eye drop at the indicated concentrations. All of the RNA was extracted from each cell, hybridised with ${ }^{32} \mathrm{P}$-labelled MYOC cDNA, and subjected to autoradiography (upper panel). RNA was also stained with ethidium bromide as a loading control (lower panel). 
Table 1 Genotype distributions and allele frequencies of (GT)n repeat markers in the $5^{\prime}$-flanking region of MYOC in glaucoma patients and normal controls

\begin{tabular}{|c|c|c|c|c|c|c|c|c|c|c|}
\hline & \multicolumn{7}{|c|}{ Genotype distribution } & \multicolumn{3}{|c|}{ Allele frequency } \\
\hline & n & $13 / 13$ & $13 / 14$ & $13 / 15$ & 14/15 & $14 / 14$ & $15 / 15$ & 13 & 14 & 15 \\
\hline ontrol & 74 & 20 & 15 & 32 & 2 & 5 & 0 & 0.588 & 0.115 & 0.297 \\
\hline POAG & 60 & 24 & 9 & 18 & 3 & 5 & 1 & 0.625 & 0.117 & 0.258 \\
\hline NTG & 47 & 25 & 3 & 14 & 0 & 4 & 1 & 0.713 & 0.053 & 0.234 \\
\hline SIG & 61 & 29 & 1 & 24 & 2 & 5 & 0 & 0.680 & 0.025 & 0.295 \\
\hline
\end{tabular}

POAG, primary open-angle glaucoma; NTG, normal tension glaucoma; SIG, steroid-induced glaucoma.

in normal control populations and patient populations are summarised in table 1 . Three alleles, $(\mathrm{G}-\mathrm{T})_{12},(\mathrm{G}-\mathrm{T})_{16}$ and (G$\mathrm{T})_{17}$, occurred so rarely that they were excluded from the analysis. The frequencies observed did not deviate significantly from those predicted by the Hardy-Weinberg equilibrium. However, there was no evidence of any differences in the genotype distributions and allele frequencies between the glaucoma groups and the control group.

In addition to the polymorphic microsatellites, we found three single nucleotide polymorphisms (SNP), G-83A, T-224C and G-306T, all of which have already been reported. ${ }^{9}$ The genotype distribution and allele frequencies of the SNPs in the normal control group and patient groups are summarised in table 2. SNP analyses also showed no evidence of association between MYOC and glaucoma; none of the three SNP sites showed an association with POAG, NTG or SIG.

\section{DISCUSSION}

Clinically, dexamethasone and prednisolone increase IOP more frequently than fluorometholone, hydrocortisone or rimexolone. ${ }^{10}$ Fluorometholone in particular is less likely to increase IOP. Rimexolone, which was recently introduced, also has a low IOP-elevating potential that is comparable to that of fluorometholone in adults. ${ }^{11}$ Nevertheless, eye drops with a low IOP-elevating potential induced MYOC to the same extent as eye drops with a high IOP-elevating potential. If $M Y O C$ induction causes IOP elevation, the potency of $M Y O C$ induction by an eye drop should correlate with the IOP elevation caused by that drug. Therefore, our results suggest that MYOC induction is not a cause of IOP elevation in SIG.

Clinically, fluorometholone and dexamethasone are used at a concentration of $0.1 \%$, whereas prednisolone and rimexolone are used at a concentration of $1 \%$. Taking this into account, the extent of MYOC induction by fluorometholone or dexamethasone is 10 times less than that shown in figure 2. This further suggests that $M Y O C$ induction is not primarily responsible for steroid-induced IOP elevation.

MYOC induction in glaucomatous eyes might be the result, rather than the cause, of IOP elevation. Initially, a clear induc- tion of $M Y O C$ expression was seen in perfused human anterior segments at high IOP. ${ }^{12}$ However, no evidence of MYOC induction was obtained in combined tissues of rat iridocorneal angles, despite IOP elevation. ${ }^{13}$ In the present study, we cultured HTM cells at a hydrostatic pressure of $32 \mathrm{~mm} \mathrm{Hg}$ above atmospheric pressure, which mimics an IOP $>32 \mathrm{~mm} \mathrm{Hg}$ in glaucoma patients. This condition stressed the cells, as demonstrated by apparent changes of cellular morphology, but did not lead to MYOC expression, suggesting that it is not a secondary response to IOP elevation. Previous studies have shown that there are no prominent differences in the signal intensities for $M Y O C$ expression between glaucomatous and non-glaucomatous eyes. ${ }^{6}$ Furthermore, no significant increase in $M Y O C$ expression was observed in the TM of rats in a steroid-induced ocular hypertension model, despite IOP elevation. ${ }^{14}$ Together, these studies support our results, arguing against the hypothesis that MYOC induction could perhaps be considered part of a protective response mechanism, rather than a homeostatic one. ${ }^{12}$

In the $5^{\prime}$-flanking region of the MYOC, we found three bi-allelic variants along with one dinucleotide repeat polymorphism, but no association between the markers with any subtype of OAG. This result was somewhat unexpected, because $M Y O C$ had been considered to be a susceptibility gene for POAG. ${ }^{1}$ Genetic studies showed that alterations in the coding region of $M Y O C$ are associated probably with a gain of pathogenic function rather than a haplo-insufficiency in the pathogenesis of glaucoma. ${ }^{15} 16$ This fact can support the lack of association between POAG and variants in the promoter region of the MYOC. It is also uncertain whether the size of this study was large enough to detect any association between the markers and POAG.

A possible association might be mediated either by a marker itself or by other polymorphisms in linkage disequilibrium with alleles of the marker. The lack of association between our markers and OAG therefore implies no evidence that the markers themselves are causative polymorphisms of OAG or under linkage disequilibrium with other authentic polymorphisms or mutations in other regions of MYOC. No published results have definitively described $M Y O C$ promoter mutations as a cause of glaucoma. ${ }^{9}{ }^{17-20}$ The T-153A polymorphism was reported to occur more frequently in Japanese POAG patients than in controls ${ }^{21}$ and the C-1000G polymorphism, also designated as MYOC.mt1, was reported to be related to the severity of POAG. ${ }^{22}{ }^{23}$ However, the former polymorphism has never been identified in other races and the C-1000G polymorphism appeared to be of no value in the evaluation of patients with glaucoma in ensuing studies. ${ }^{9} 2425 \mathrm{It}$ appears that a predisposition to glaucoma related to $M Y O C$ is mediated through structural gene alterations, but not by noncoding sequence variants such as promoter sequence variants. Studies on transgenic mice showed that mice overexpressing $M Y O C$ at a level similar to that induced by steroid use did not

Table 2 Genotype distributions and allele frequencies of polymorphic variants in the 5 -flanking region of MYOC in glaucoma patients and normal controls

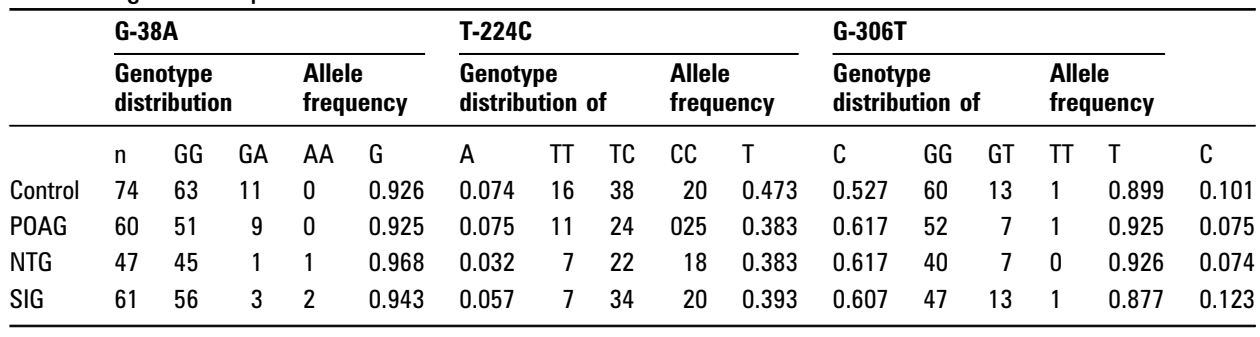

POAG, primary open-angle glaucoma; NTG, normal tension glaucoma; SIG, steroid-induced glaucoma. 
develop elevated IOP or glaucoma, ${ }^{78}$ suggesting that increased $M Y O C$ expression alone is not sufficient to cause glaucoma. If the level of $M Y O C$ is not important in determining the glaucoma phenotype, it is likely that any promoter sequence variant is not directly involved in the development of OAG. This could be an explanation for the lack of association between our markers and OAG. Several genes were mapped to GLC1A but excluded as candidates for OAG by genetic linkage analysis. ${ }^{1}$ Therefore, we do not believe that our SNPs are under linkage disequilibrium with other genes that may be responsible for the development of glaucoma.

In summary, we investigated whether MYOC induction can lead to IOP elevation or vice versa and if any $M Y O C$ promoter variant is associated with OAG. We showed that MYOC expression in vitro was neither correlated with elevated IOP in vivo nor induced by hydrostatic pressure mimicking elevated IOP. We also showed that the MYOC gene itself is not associated with OAG, including POAG, NTG and SIG. Therefore, our results do not support the hypothesis that $M Y O C$ induction might be linked to IOP variation and that promoter variants of MYOC could be a risk factor for the pathogenesis of OAG.

Funding This work was supported by the Korea Research Foundation Grant funded by the Korean Government (MOEHRD, Basic Research Promotion Fund) (KRF-2004-041-E00216), by a grant from the Korea Science and Engineering Foundation Science Research Center (SRC) project endowed to the Molecular Therapeutic Research Center (MTRC) and by Samsung Biomedical Research institute grant \#SBRI C-A7-402-1.

Competing interests None.

Patient consent Obtained.

Provenance and peer review Not commissioned; externally peer reviewed.

\section{REFERENCES}

1. Stone EM, Fingert JH, Alward WL, et al. Identification of a gene that causes primary open angle glaucoma. Science 1997;275:668-70.

2. Nguyen TD, Chen P, Huang WD, et al. Gene structure and properties of TIGR, an olfactomedin-related glycoprotein cloned from glucocorticoid-induced trabecular meshwork cells. J Biol Chem 1998;273:6341-50.

3. Francois J. Cortisone et tension ocularie. Ann Ocul (Paris) 1954;187:805-16.

4. Lutjen-Drecoll E, May CA, Polansky JR, et al. Localization of the stress proteins alpha B-crystallin and trabecular meshwork inducible glucocorticoid response protein in normal and glaucomatous trabecular meshwork. Invest Ophthalmol Vis Sci 1998;39:517-25.

5. Fautsch MP, Bahler CK, Jewison DJ, et al. Recombinant TIGR/MYOC increases outflow resistance in the human anterior segment. Invest Ophthalmol Vis Sci 2000:41:4163-8.
6. Wang $\mathbf{X}$, Johnson DH. mRNA in situ hybridization of TIGR/MYOC in human trabecular meshwork. Invest Ophthalmol Vis Sci 2000;41:1724-9.

7. Gould DB, Miceli-Libby L, Savinova OV, et al. Genetically increasing Myoc expression supports a necessary pathologic role of abnormal proteins in glaucoma. Mol Cell Biol 2004;24:9019-25.

8. Zillig M, Wurm A, Grehn FJ, et al. Overexpression and properties of wild-type and Tyr437His mutated myocilin in the eyes of transgenic mice. Invest Ophthalmol Vis Sci 2005; 46:223-34

9. Fan BJ, Leung YF, Pang CP, et al. Polymorphisms in the myocilin promoter unrelated to the risk and severity of primary open-angle glaucoma. J Glaucoma 2004; 13:377-84

10. Mindel JS, Tavitian HO, Smith H Jr, et al. Comparative ocular pressure elevation by medrysone, fluorometholone, and dexamethasone phosphate. Arch Ophthalmol 1980;98:1577-8

11. Leibowitz HM, Bartlett JD, Rich R, et al. Intraocular pressure-raising potential of $1.0 \%$ rimexolone in patients responding to corticosteroids. Arch Ophthalmol 1996;114:933-7.

12. Borras T, Rowlette LL, Tamm ER, et al. Effects of elevated intraocular pressure on outflow facility and TIGR/MYOC expression in perfused human anterior segments. Invest Ophthalmol Vis Sci 2002:43:33-40.

13. Ahmed F, Torrado M, Johnson E, et al. Changes in mRNA levels of the Myoc/Tigr gene in the rat eye after experimental elevation of intraocular pressure or optic nerve transection. Invest Ophthalmol Vis Sci 2001:42:3165-72.

14. Sawaguchi K, Nakamura Y, Sakai H, et al. Myocilin gene expression in the trabecular meshwork of rats in a steroid-induced ocular hypertension model. Ophthalmic Res 2005:37:235-42.

15. Kim BS, Savinova OV, Reedy MV, et al. Targeted disruption of the Myocilin Gene (Myoc) suggests that human glaucoma-causing mutations are gain of function. $\mathrm{Mol}$ Cell Biol 2001;21:7707-13.

16. Wiggs JL, Vollrath D. Molecular and clinical evaluation of a patient hemizygous for TIGR/MYOC. Arch Ophthalmol 2001:119:1674-8.

17. Fingert JH, Clark AF, Craig JE, et al. Evaluation of the myocilin (MYOC) glaucoma gene in monkey and human steroid-induced ocular hypertension. Invest Ophthalmol Vis Sci 2001:42:145-52.

18. Sjostrand A, Tomic L, Larsson $\mathrm{LI}$, et al. No evidence of association between GT/CA repeat polymorphism in the GLC1A gene promoter and primary open-angle or exfoliation glaucoma. Acta Ophthalmol Scand 2002;80:384-6.

19. Saura M, Cabana M, Ayuso C, et al. Mutations including the promoter region of myocilin/TIGR gene. Eur J Hum Genet 2005:13:384-7.

20. Lopez-Martinez F, Lopez-Garrido MP, Sanchez-Sanchez F, et al. Role of MYOC and OPTN sequence variations in Spanish patients with primary open-angle glaucoma. $\mathrm{Mol}$ Vis 2007:13:862-72.

21. Suzuki R, Hattori Y, Okano K. Promoter mutations of myocilin gene in Japanese patients with open angle glaucoma including normal tension glaucoma. $\mathrm{Br} \mathrm{J}$ Ophthalmol 2000:84:1078

22. Colomb E, Nguyen TD, Bechetoille A, et al. Association of a single nucleotide polymorphism in the TIGR/MYOCILIN gene promoter with the severity of primary open-angle glaucoma. Clin Genet 2001;60:220-5.

23. Polansky JR, Juster RP, Spaeth GL. Association of the myocilin mt.1 promoter variant with the worsening of glaucomatous disease over time. Clin Genet 2003;64:18-27.

24. Alward WL, Kwon $\mathrm{YH}$, Khanna $\mathrm{CL}$, et al. Variations in the myocilin gene in patients with open-angle glaucoma. Arch Ophthalmol 2002;120:1189-97.

25. Ozgul RK, Bozkurt B, Orcan S, et al. Myocilin mt1 promoter polymorphism in Turkish patients with primary open angle glaucoma. Mol Vis 2005:11:916-21. 